\title{
On the Integral Giving the Degree of a Map and a Rouché Type Theorem
}

\author{
T. Hatziafratis and A. Tsarpalias
}

\begin{abstract}
An analytic approach to the degree of a map $f: \partial D \longrightarrow \mathbb{R}^{n} \backslash\{0\}$ is given (where $D \subset \mathbb{R}^{n}$ is a bounded domain with smooth boundary) and a Rouché type theorem is proved.
\end{abstract}

Keywords: Degree of a map, differential forms, fixed points

AMS subject classification: Primary $58 \mathrm{~A} 10$, secondary $58 \mathrm{C} 30$

\section{Introduction}

In this paper we give a simple analytic proof that the integral

$$
\frac{\Gamma(n / 2)}{2 \pi^{n / 2}} \int_{\partial D} \frac{1}{|f|^{n}} \sum_{j=1}^{n}(-1)^{j-1} f_{j} d f_{1} \wedge \cdots(j) \cdots \wedge d f_{n}
$$

is an integer for a continuously differentiable map

$$
f: \partial D \longrightarrow \mathbb{R}^{n} \backslash\{0\},
$$

where $D$ is a bounded domain in $\mathbb{R}^{n}$ with smooth boundary; this integral gives the degree of $f$.

The basic idea of this proof is the following: if we call $\eta(f)$ the integrand in the above integral then, although $\eta(f)$ is not, in general, $d$-exact on the $(n-1)$-dimensional manifold $\partial D$, its derivative

$$
\frac{\partial \eta(f(\cdot, t))}{\partial t}
$$

is $d$-exact, when we let $f$ depend on a parameter $t$; and this is proved by constructing explicitly a $d$-primitive (see Lemma 2). As for the parametrization of $f$, it is done with a perturbation argument, based on Sard's theorem. For another analytic proof see Heinz [2]. For the history of the above integral as well as its connections with polynomial equations and the Gauss-Bonnet theorem see Siegberg [3]. We also prove a version of Rouché's principle which gives a proof of Brouwer's fixed point theorem. A special case of it is contained in [5].

T. Hatziafratis: University of Athens, Dept. Math., Panepistimiopolis, 15784 Athens, Greece

A. Tsarpalias: University of Athens, Dept. Math., Panepistimiopolis, 15784 Athens, Greece 


\section{Preliminaries}

Let us recall some basic facts about differential forms in $\mathbb{R}^{n}$. A $p$-form in an open set $G$ in $\mathbb{R}^{n}$ is a function $\omega$, symbolically represented by the sum

$$
\omega=\sum_{1 \leq i_{1}, \ldots, i_{p} \leq n} f_{i_{1}, \ldots, i_{p}}(x) d x_{i_{1}} \wedge \cdots \wedge d x_{i_{p}}
$$

which assigns to each smooth $p$-surface $\mathcal{X}$ in $G$ a number $\omega(\mathcal{X})=\int_{\mathcal{X}} \omega$ according to the rule

$$
\int_{\mathcal{X}} \omega=\sum \int_{\Omega} f_{i_{1}, \ldots, i_{p}}(\mathcal{X}(t)) \frac{\partial\left(x_{i_{1}}, \ldots, x_{i_{p}}\right)}{\partial\left(t_{1}, \ldots, t_{p}\right)} d t_{1} \cdots d t_{p}
$$

where $\Omega$ is the parameter domain of $\mathcal{X}$, and $f_{i_{1}, \ldots, i_{p}}$ are real $C^{1}$-functions on $G$. The number $\int_{\mathcal{X}} \omega$ is called also the integral of $\omega$ on $\mathcal{X}$. The algebra of differential forms obeys the laws of exterior algebra. We recall that a basic rule of exterior algebra is the general anticommutative law:

$$
d x_{j_{1}} \wedge \cdots \wedge d x_{j_{p}}=\operatorname{sign}\left(\begin{array}{l}
i_{1} \cdots i_{p} \\
j_{1} \cdots j_{p}
\end{array}\right) d x_{i_{1}} \wedge \cdots \wedge d x_{i_{p}}
$$

where $\left(j_{1} \cdots j_{p}\right)$ is a permutation of $\left(i_{1} \cdots i_{p}\right)$ and $\operatorname{sign}\left(\begin{array}{l}i_{1} \cdots i_{p} \\ j_{1} \cdots j_{p}\end{array}\right)$ is the sign of the permutation. Also the differentiation of differential forms is done according to the rule

$$
d \omega=\sum d f_{i_{1}, \ldots, i_{p}}(x) \wedge d x_{i_{1}} \wedge \cdots \wedge d x_{i_{p}}
$$

where $d f=\sum \frac{\partial f}{\partial x_{i}} d x_{i}$.

With the above terminology and notation Stokes' theorem takes the following form.

Stokes' Theorem. If $D \subset \mathbb{R}^{n}$ is an open bounded set with smooth boundary $\partial D$, and $\omega$ is an $(n-1)$-form in a neighborhood of $\bar{D}$, then

$$
\int_{D} d \omega=\int_{\partial D} \omega
$$

We will use determinants with entries differential forms: if $a_{i j}$ are differential forms, then

$$
\operatorname{det}\left[\begin{array}{ccc}
a_{11} & \cdots & a_{n 1} \\
\vdots & & \vdots \\
a_{1 n} & \cdots & a_{n n}
\end{array}\right]=\operatorname{det}\left[a_{1 j}, \ldots, a_{n j}\right]=: \sum_{\sigma} \operatorname{sign}(\sigma) a_{1 \sigma(1)} \wedge \cdots \wedge a_{n \sigma(n)}
$$

where the summation is extended over all permutations $\sigma$ of $\{1, \ldots, n\}$. (The elements of each column are assumed to be differential forms of the same degree; this degree may change from column to column.) Thus when we write $\operatorname{det}\left[a_{1}, \ldots, a_{n}\right]$, we mean that $j$ runs from 1 to $n$ forming the $n$ rows of the determinant. The value of such a determinant does not change if we add to a row a multiple of another row (we mean multiplied by a function). In some determinants a column may be repeated, and we 
put an index to indicate how many times this column is repeated. For example, in the determinant

$$
\operatorname{det}_{n-2}\left[\begin{array}{ccccc}
a_{11} & a_{12} & b_{1} & \cdots & b_{1} \\
\vdots & \vdots & \vdots & & \vdots \\
a_{n 1} & a_{n 2} & b_{n} & \cdots & b_{n}
\end{array}\right]
$$

the column $\left[b_{1}, \ldots, b_{n}\right]^{T}$ is repeated $n-2$ times. A determinant of the form

$$
\operatorname{det}_{n-1}\left[\begin{array}{cccc}
a_{1} & b_{1} & \cdots & b_{1} \\
\vdots & \vdots & & \vdots \\
a_{n} & b_{n} & \cdots & b_{n}
\end{array}\right]
$$

is denoted by $\operatorname{det}_{1, n-1}\left[a_{j}, b_{j}\right]$. Now if the $a_{j}$ are functions and the $b_{j}$ are 1 -forms then, expanding the above determinant, we obtain

$$
\operatorname{det}_{1, n-1}\left[a_{j}, b_{j}\right]=(n-1) ! \sum_{j=1}^{n}(-1)^{j-1} a_{j} \cdot b_{1} \wedge \cdots(j) \cdots \wedge b_{n} .
$$

If furthermore $d b_{j}=0$, then

$$
d\left(\operatorname{det}_{1, n-1}\left[a_{j}, b_{j}\right]\right)=\operatorname{det}_{1, n-1}\left[d a_{j}, b_{j}\right] .
$$

All these properties follow from the corresponding properties of the usual determinants if we take into consideration the anticommutative law for differential forms:

$$
\omega \wedge \eta=(-1)^{p q} \eta \wedge \omega
$$

where

$$
p=\operatorname{deg} \omega \quad \text { and } \quad q=\operatorname{deg} \eta .
$$

For more properties of such determinants see [1: p. 8] and for the calculus of differential forms, that we are using, see [4: Chapter 4].

Deflnition. Let $D \subset \mathbb{R}^{n}$ be a bounded domain with smooth boundary. For a $C^{1}$-map $f: \partial D \longrightarrow \mathbb{R}^{n} \backslash\{0\}$ we define

$$
\eta(f)=\frac{c}{|f|^{n}} \sum_{j=1}^{n}(-1)^{j-1} f_{j} d f_{1} \wedge \cdots(j) \cdots \wedge d f_{n}
$$

where $|f|^{2}=\sum_{j=1}^{n} f_{j}^{2}$ and $c=\frac{\Gamma(n / 2)}{2 \pi^{n / 2}}$. The degree $\delta(f)$ of $f$ is defined by the integral

$$
\delta(f)=\int_{\partial D} \eta(f)
$$




\section{The main results}

We start be proving some lemmas from which the main results, Theorems 1 and 2, will follow; Lemma 2 is the main step.

Lemma 1. Let $D \subset \mathbb{R}^{n}$ be a domain and $f: \partial D \longrightarrow \mathbb{R}^{n} \backslash\{0\}$ a $C^{2} \cdot \operatorname{map}$. Then $d \eta(f)=0$ in $D$, and for a $C^{2}$-function $\phi: D \longrightarrow(0, \infty)$ we have $\eta(\phi \cdot f)=\eta(f)$.

Proof. Let us notice that

$$
\eta(f)=\frac{c^{\prime}}{|f|^{n}} \operatorname{det}_{1, n-1}\left[f_{j}, d f_{j}\right]
$$

where $c^{\prime}=\frac{c}{(n-1) !}$. Therefore,

$$
\begin{aligned}
\eta(\phi \cdot f) & =\frac{c^{\prime}}{\phi^{n}|f|^{n}} \operatorname{det}_{1, n-1}\left[\phi f_{j}, \phi d f_{j}+f_{j} d \phi\right] \\
& =\frac{c^{\prime}}{\phi^{n-1}|f|^{n}} \operatorname{det}_{1, n-1}\left[f_{j}, \phi d f_{j}+f_{j} d \phi\right] \\
& =\frac{c^{\prime}}{\phi^{n-1}|f|^{n}} \operatorname{det}_{1, n-1}\left[f_{j}, \phi d f_{j}\right] \\
& =\frac{c^{\prime}}{\phi^{n}|f|^{n}} \operatorname{det}_{1, n-1}\left[f_{j}, d f_{j}\right] \\
& =\eta(f)
\end{aligned}
$$

which proves the second assertion of the lemma; for the first assertion apply what we have just proved with $\phi=|f|^{-1}$ in order to obtain

$$
\eta(f)=\eta(g)=c^{\prime} \operatorname{det}_{1, n-1}\left[g_{j}, d g_{j}\right]
$$

where $g=|f|^{-1} f$. Then

$$
d \eta(f)=d \eta(g)=c^{\prime} \operatorname{det}_{1, n-1}\left[d g_{j}, d g_{j}\right]=c^{\prime} n ! d g_{1} \wedge \cdots \wedge d g_{n} .
$$

But $\sum_{j=1}^{n} g_{j}^{2}=1$ and therefore $\sum_{j=1}^{n} g_{j} d g_{j}=0$ which implies $d g_{1} \wedge \cdots \wedge d g_{n}=0$, i.c. $d \eta(f)=0$

Lemma 2. If $f=f(x, t):(\partial D) \times[0,1] \longrightarrow \mathbb{R}^{n} \backslash\{0\}$ is a $C^{2}$-map, then the differential form $\frac{\partial}{\partial t} \eta(f(\cdot, t))$ is d-exact; more precisely,

$$
\frac{\partial}{\partial t} \eta(f(\cdot, t))=d_{x} \theta
$$

where

$$
\theta=\frac{(n-1) \cdot c^{\prime}}{|f|^{n}} \operatorname{det}_{n-2}\left[\begin{array}{ccccc}
f_{1} & f_{1, t} & d f_{1} & \cdots & d f_{1} \\
\vdots & \vdots & \vdots & & \vdots \\
f_{n} & f_{n, t} & d f_{n} & \cdots & d f_{n}
\end{array}\right]
$$


with $f_{j, t}=\frac{\partial f_{j}}{\partial t}$.

Proof. Exactly as in the case of $\eta$ (see Lemma 1), $\theta$ remains the same if $f$ is multiplied by a $C^{2}$-function

$$
\phi(x, t):(\partial D) \times[0,1] \longrightarrow(0, \infty) .
$$

Hence we may assume, without loss of generality, that

$$
\sum_{j=1}^{n} f_{j}^{2}(x, t)=1
$$

We may also assume that, near the point at which we want to prove $(1), f_{1} \neq 0$. Then

$$
f_{1} \eta(f(\cdot, t))=c^{\prime} \operatorname{det}_{n-1}\left[\begin{array}{cccc}
f_{1}^{2} & f_{1} d f_{1} & \cdots & f_{1} d f_{1} \\
f_{2} & d f_{2} & \cdots & d f_{2} \\
\vdots & \vdots & & \vdots \\
f_{n} & d f_{n} & \cdots & d f_{n}
\end{array}\right]
$$

(Throughout this proof $d=d_{x}$.) Now multiplying each $j$-th row of this determinant by $f_{j}(2 \leq j \leq n)$ and adding them to the first row we obtain, in view of (2),

$$
f_{1} \eta(f(\cdot, t))=c^{\prime} \operatorname{det}_{n-1}\left[\begin{array}{cccc}
1 & 0 & \cdots & 0 \\
f_{2} & d f_{2} & \cdots & d f_{2} \\
\vdots & \vdots & & \vdots \\
f_{n} & d f_{n} & \cdots & d f_{n}
\end{array}\right]=c d f_{2} \wedge \cdots \wedge d f_{n}
$$

Hence

and therefore

$$
\eta(f(\cdot, t))=\frac{c}{f_{1}} d f_{2} \wedge \cdots \wedge d f_{n}
$$

$$
\frac{\partial}{\partial t} \eta(f(\cdot, t))=-c \frac{f_{1, t}}{f_{1}^{2}} d f_{2} \wedge \cdots \wedge d f_{n}+\frac{c}{f_{1}} \sum_{j=2}^{n} d f_{2} \wedge \cdots \wedge d f_{j, t} \wedge \cdots \wedge d f_{n}
$$

On the other hand, as a similar computation shows,

$$
\begin{aligned}
f_{1} \cdot \theta & =(n-1) c^{\prime} \operatorname{det}_{n-2}\left[\begin{array}{ccccc}
f_{1}^{2} & f_{1} f_{1, t} & f_{1} d f_{1} & \cdots & f_{1} d f_{1} \\
f_{2} & f_{2, t} & d f_{2} & \cdots & d f_{2} \\
\vdots & \vdots & \vdots & & \vdots \\
f_{n} & f_{n, t} & d f_{n} & \cdots & d f_{n}
\end{array}\right] \\
= & {\left[\begin{array}{ccccc}
1 & 0 & 0 & 0 & 0 \\
f_{2} & f_{2, t} & d f_{2} & \cdots & d f_{2} \\
\vdots & \vdots & \vdots & & \vdots \\
f_{n} & f_{n, t} & d f_{n} & \cdots & d f_{n}
\end{array}\right] } \\
= & {\left[\begin{array}{cccc}
f_{2, t} & d f_{2} & \cdots & d f_{2} \\
\vdots & \vdots & & \vdots \\
f_{n, t} & d f_{n} & \cdots & d f_{n}
\end{array}\right] }
\end{aligned}
$$


Hence

$$
\theta=\frac{(n-1) c^{\prime}}{f_{1}} \operatorname{det}_{n-2}\left[\begin{array}{cccc}
f_{2, \ell} & d f_{2} & \cdots & d f_{2} \\
\vdots & \vdots & & \vdots \\
f_{n, t} & d f_{n} & \cdots & d f_{n}
\end{array}\right]
$$

and therefore

$$
\begin{aligned}
d \theta= & -\frac{(n-1) c^{\prime}}{f_{1}^{2}} d f_{1} \wedge \operatorname{det}_{n-2}\left[\begin{array}{cccc}
f_{2, t} & d f_{2} & \cdots & d f_{2} \\
\vdots & \vdots & & \vdots \\
f_{n, t} & d f_{n} & \cdots & d f_{n}
\end{array}\right] \\
& +\frac{(n-1) c^{\prime}}{f_{1}} \operatorname{det}_{n-2}\left[\begin{array}{cccc}
d f_{2, \ell} & d f_{2} & \cdots & d f_{2} \\
\vdots & \vdots & & \vdots \\
d f_{n, t} & d f_{n} & \cdots & d f_{n}
\end{array}\right]
\end{aligned}
$$

Now (2) gives

$$
f_{1} d f_{1}=-\sum_{j=2}^{n} f_{j} d f_{j} \quad \text { and } \quad f_{1} f_{1, t}=-\sum_{j=2}^{n} f_{j} f_{j, t}
$$

Hence

$$
\begin{aligned}
f_{1} d f_{1} \wedge & \operatorname{det}_{n-2}\left[\begin{array}{cccc}
f_{2, t} & d f_{2} & \cdots & d f_{2} \\
\vdots & \vdots & & \vdots \\
f_{n, t} & d f_{n} & \cdots & d f_{n}
\end{array}\right] \\
& =\left(-\sum_{j=2}^{n} f_{j} d f_{j}\right) \wedge \operatorname{det}_{n-2}\left[\begin{array}{cccc}
f_{2, t} & d f_{2} & \cdots & d f_{2} \\
\vdots & \vdots & & \vdots \\
f_{n, t} & d f_{n} & \cdots & d f_{n}
\end{array}\right] \\
& =-(n-2) !\left(\sum_{j=2}^{n} f_{j} d f_{j}\right) \wedge\left(\sum_{j=2}^{n}(-1)^{j} f_{j, t} d f_{2} \wedge \cdots(j) \cdots \wedge d f_{n}\right) \\
& =-(n-2) !\left(\sum_{j=2}^{n} f_{j} f_{j, t}\right) d f_{2} \wedge \cdots \wedge d f_{n} \\
& =(n-2) ! f_{1} f_{1, t} d f_{2} \wedge \cdots \wedge d f_{n} .
\end{aligned}
$$

Also,

$$
\operatorname{det}_{n-2}\left[\begin{array}{cccc}
d f_{2, t} & d f_{2} & \cdots & d f_{2} \\
\vdots & \vdots & & \vdots \\
d f_{n, t} & d f_{n} & \cdots & d f_{n}
\end{array}\right]=(n-2) \sum_{j=2}^{n} d f_{2} \wedge \cdots \wedge d f_{j, t} \wedge \cdots \wedge d f_{n}
$$

Substituting (5) and (6) into (4) and comparing with (3), we obtain (1). The proof is complete 
Lemma 3. If $f=f(x, t):(\partial D) \times[0,1] \rightarrow \mathbb{R}^{n} \backslash\{0\}$ is a $C^{2}$-map, then the integral is independent of $t$.

$$
\delta(f(\cdot, t))=\int_{\partial D} \eta(f(\cdot, t))
$$

Proof. In view of Lemma 2 and Stokes' theorem, we obtain

$$
\frac{\partial}{\partial t} \delta(f(\cdot, t))=\int_{\partial D} \frac{\partial}{\partial t} \eta(f(\cdot, t))=\int_{\partial D} d_{x} \theta=\int_{\partial(\partial D)} \theta=0
$$

and the assertion of the lemma follows

Lemma 4. Let $f: \bar{D} \longrightarrow \mathbb{R}^{n}$ be a $C^{1}$-map with $f(x) \neq 0$ for all $x \in \partial D$. Then for $\varepsilon>0$ there is an $y \in \mathbb{R}^{n}$ with $|y|<\varepsilon$ so that the equation $f(x)=y$ has at most a finite number of solutions, say $x^{1}, \ldots, x^{p} \in D$, at which $J_{f}\left(x^{i}\right) \neq 0$ for $i=1, \ldots, p$ (here $J_{f}$ denotes the Jacobian of $f$ ) and such that $f(x) \neq y$ for all $x \in \partial D$.

Proof. Let $C=\left\{x \in D: J_{f}(x)=0\right\}$. Then, by Sard's theorem, the set $f(C)$, the image of $C$ under $f$, has measure zero. Hence there is an $y \in \mathbb{R}^{n}$ with $|y|<\varepsilon$ so that $y \notin f(C)$. Since $f(x) \neq 0$ for all $x \in \partial D$, we may also choose $y$ sufficiently close to 0 so that $f(x) \neq y$ for all $x \in \partial D$.

We claim that the equation $f(x)=y$ has at most a finite number of solutions; for otherwise there would exist a sequence $\left\{x^{\nu}\right\}_{\nu \geq 1} \subset D$ of distinct points with

$$
x^{\nu} \rightarrow x \in \bar{D} \text { as } \nu \rightarrow \infty \quad \text { and } \quad f\left(x^{\nu}\right)=y \text { for all } \nu \geq 1 \text {. }
$$

But then $f(x)=y$ whence $x \in D$ and $J_{f}(x) \neq 0$. Therefore, by the inverse function theorem, $f$ is one-to-one on a neighborhood of $x$, which contradicts (7) and the proof is complete

Lemma 5. Suppose that $f: U \longrightarrow \mathbb{R}^{n}$ is a $C^{2}$-map on an open set $U \subset \mathbb{R}^{n}$ such that $f$ is a diffeomorphism on a neighborhood of a point a $\in U$ to a neighborhood of $b=f(a)$. Then

$$
\int_{\partial B(a, \varepsilon)} \eta(f-b)=1 \quad \text { or } \quad \int_{\partial B(a, \varepsilon)} \eta(f-b)=-1
$$

for sufficiently small $\varepsilon>0$, where $B(x, \varepsilon)=\left\{y \in \mathbb{R}^{n}:|y-x|<\varepsilon\right\}$.

Proof. We have

$$
\int_{\partial B(a, \varepsilon)} \eta(f-b)=c \int_{f(B(a, c))} \frac{1}{|y-b|^{n}} \sum_{j=1}^{n}(-1)^{j-1}\left(y_{j}-b_{j}\right) d y_{1} \wedge \cdots(j) \cdots \wedge y_{n}
$$

But, by Lemma 1 and Stokes' theorem, the above integral becomes

$$
\delta(f)=c \int_{\partial B(0,1)} \sum_{j=1}^{n}(-1)^{j-1} y_{j} d y_{1} \wedge \cdots(j) \cdots \wedge d y_{n}
$$

(here $\partial B(0,1)$ could have either orientation depending upon $f$ ). Therefore, by Stokes' theorem again,

$$
\delta(f)= \pm c \int_{B(0,1)} n d y_{1} \wedge \cdots \wedge d y_{n}= \pm c \operatorname{Vol}(B(0,1))= \pm 1
$$

and the proof is complete 
Theorem 1. Let $D \subset \mathbb{R}^{n}$ be a bounded domain with smooth boundary and $f$ : $\partial D \longrightarrow \mathbb{R}^{n} \backslash\{0\}$ a $C^{1}$-map. Then $\delta(f)$ is an integer.

Proof. Let us assume first that $f$ is a $C^{2}$-map and let $\tilde{f}: \bar{D} \rightarrow \mathbb{R}^{n}$ be a $C^{2}$. extension of $f$. By Lemma 4 there is an $y \in \mathbb{R}^{n}$, sufficiently close to zero so that the equation $\tilde{f}(x)=y$ has finitely many solutions $x^{1}, \ldots, x^{p} \in D$ with $J_{f}\left(x^{i}\right) \neq 0$ and $f(x) \neq u$ for $x \in \partial D$ and $|u| \leq|y|$.

But, by Lemma $3, \delta(f)=\delta(f-y)$. Also, for small $\varepsilon>0$,

$$
\delta(f-y)=\int_{\partial D} \eta(f-y)=\sum_{i=1}^{p} \int_{\partial B\left(x^{i}, \varepsilon\right)} \eta(\tilde{f}-y)
$$

where $B\left(x^{i}, \varepsilon\right)=\left\{x \in \mathbb{R}^{n}:\left|x-x^{i}\right| \leq \varepsilon\right\} ;$ the last equation follows from Stokes' theorem applied to $\eta(\tilde{f}-y)$ and the domain

$$
\Omega=D \backslash \bigcup_{i=1}^{p} B\left(x^{i}, \varepsilon\right)
$$

since $d \eta(\tilde{f}-y)=0$ by Lemma 1 .

But $\tilde{f}-y$ is a $C^{2}$-diffeomorphism on a neighbothood of $x^{i}$ and therefore, if $\varepsilon$ is sufficiently small,

$$
\int_{\partial B\left(x^{i}, \varepsilon\right)} \eta(\tilde{f}-y)= \pm 1
$$

by Lemma 5 . Hence $\delta(f-y)$ is an integer and so is $\delta(f)$. This completes the proof in the case $f$ is of class $C^{2}$. If $f$ is only of class $C^{1}$, then we can approximate $f$ by $C^{2}$-functions, uniformly on $\partial D$ and prove that, in this case too, $\delta(f)$ is an integer. This completes the proof $\mathbf{B}$

Theorem 2. Let $D \subset \mathbb{R}^{n}$ be a bounded domain with smooth boundary. Suppose that $f: \bar{D} \longrightarrow \mathbb{R}^{n}$ is a continuous map and $g: \bar{D} \longrightarrow \mathbb{R}^{n}$ is a $C^{1}$-map with $g(x) \neq 0$ for all $x \in \partial D$ and $\delta(g) \neq 0$. If $|f(x)| \leq|g(x)|$ for every $x \in \partial D$, then the equation $f(x)+g(x)=0$ has at least one solution $x$ in $\bar{D}$.

Proof. Let us prove the statement first in the case $f$ is of class $C^{1}$ and $|f(x)|<$ $|g(x)|$ for every $x \in \partial D$. Then for $t \in[0,1]$ and $x \in \partial D$ we have

$$
|g(x)+t f(x)| \geq|g(x)|-t|f(x)|>0 .
$$

Therefore $\delta(g+t f)$ is independent of $t$, since it is continuous in $t$ and integer-valued (by Theorem 1). Hence $\delta(0)=\delta(1)$, i.e. $\delta(g)=\delta(f+g)$ and therefore $\delta(f+g) \neq 0$. But if $f(x)+g(x) \neq 0$ for all $x \in \bar{D}$, then

$$
\delta(f+g)=\int_{\partial D} \eta(f+g)=0
$$

by Lemma 1 and Stokes' theorem, since $d[\eta(f+g)]=0$ in $\bar{D}$. This is a contradiction which completes the proof in this case. 
Now we consider the general case in which $f$ is only continuous and $|f(x)| \leq|g(x)|$ for $x \in \partial D$. Then, by the Stone-Weierstrass theorem, for each $\lambda \in(0,1)$ there is a sequence of polynomials $p_{\nu}$ so that

$$
p_{\nu} \rightarrow \lambda f \quad \text { uniformly on } \bar{D} \text { and }\left|p_{\nu}\right|<|g| \text { on } \bar{D} \text {. }
$$

Thus, by the first case considered, there is $x^{\nu} \in \bar{D}$ so that $p_{\nu}\left(x^{\nu}\right)+g\left(x^{\nu}\right)=0$. Passing to a subsequence, we may assume that $x^{\nu} \rightarrow x_{\lambda} \in \bar{D}$. Therefore

$$
\lambda f\left(x_{\lambda}\right)+g\left(x_{\lambda}\right)=0 .
$$

Finally, choosing $\lambda_{j} \rightarrow 1$ so that $x_{\lambda_{j}} \rightarrow x \in \bar{D}$, we obtain that $f(x)+g(x)=0$ and the proof is complete

The following immediate consequence of Theorem 2 is Brouwer's fixed point theorem. It follows from Theorem 2 by applying it with $\bar{D}$ the closed unit ball of $\mathbb{R}^{n}$ and $g(x)=-x$.

Corollary. Let $\mathbf{B}^{n}=\left\{x \in \mathbb{R}^{n}:|x| \leq 1\right\}$. Every continuous map $f: \mathbf{B}^{n} \longrightarrow \mathbf{B}^{n}$ has a fixed point.

\section{References}

[1] Aizenberg, I. and A. Yuzhakov: Integral Representations and Residues in Multidimensional Complex Analysis (Transl. Math. Monographs: Vol. 58). Providence (R. I.): Amer. Math. Soc. 1983.

[2] Heinz, E.: An elementary analytic theory of the degree of mapping in $n$-dimensional space. J. Math. Mech. 8 (1995), $231-247$.

[3] Siegberg, H.W.: Some historical remarks concerning degree theory. Amer. Math. Monthly 88 (1981), $125-139$.

[1] Spivak, M.: Calculus on Manifolds. New York: Benjarnin 1965.

[5] Tsarpalias, A.: A version of Rouchés theorem for continuous functions. Amer. Math. Monthly 96 (1989), $911-913$. 